\title{
DIRTY AND CLEAN TECHNOLOGIES
}

\author{
SUPRATIM DAS GUPTA* \\ University of South Carolina, Columbia, South Carolina; and the University of Guanajuato, Guanajuato, Mexico
}

\begin{abstract}
Pollution from fossil fuel use is a global problem. Studies have shown that a worsening of environmental quality has adverse effects on worker productivity and health. In this study, there is an inexhaustible natural resource that deteriorates environmental quality and affects productivity. There also exists a perfect substitute clean backstop, which is initially too costly to operate and whose costs can be reduced through investments in knowledge. Depending on the endowment of environmental quality, the optimal solution shows that the planner should only use the resource or only the backstop until a constant steady state is reached in which the polluting resource and backstop are used in fixed proportions. We show that investments in alternative technologies from the very beginning can help an economy make the eventual switch to clean energy sources, thereby attaining better environmental quality.
\end{abstract}

Keywords. clean technologies, dirty technologies, environmental quality, investment, knowledge stock

JEL Classifications. O32, O44, Q42, Q56, Q57

\section{Introduction}

Efforts to use renewable alternative technologies (e.g., wind, solar ${ }^{1}$ ) at a greater scale have been adopted by countries in recent years. This has been the case as rising pollution concerns (global warming, climate change) from use of fossil fuels have gained relatively more importance than fears of running out of these essential resources. In a model of total energy production from a dirty resource and a perfect substitute clean technology (backstop), we show the optimal solution implies using either one energy source at first before finally converging to a steady state of using both the dirty and clean technologies in fixed proportions. The dirty technology deteriorates environmental quality, and the average cost of

I thank two anonymous referees for their valuable comments. I am deeply indebted to my Ph.D. dissertation committee comprising John McDermott, Christian Jensen, and Jason Murray for their support throughout this project. All remaining errors are mine.

*Email: dasgupta@ugto.org

1 By "alternative" we mean cleaner technologies. Other examples for alternative renewable technologies are hydro and biomass. Examples for nonrenewable alternative technologies are nuclear energy and natural gas. 
the initially costlier clean technology falls with investments in knowledge. ${ }^{2}$ In our model, we show the social planner uses either one of the two technologies (but always invests in the clean technology) depending on the relative magnitude of the pollution cost due to environmental degradation and the average cost of the clean backstop until reaching the steady state. We arrive at the interesting result that in a situation of a stock of low environmental quality (or high pollution cost), it is optimal to use only the expensive clean technology initially if the external pollution cost of the dirty technology is correctly taken into account. A case can be made for a developing country with a high pollution level where using a costlier backstop (but with continuous investments in knowledge to reduce its average cost as in Tsur and Zemel [2003]) would help improve environmental quality, followed by, at a high enough level of the environment, a switch to dual use of the dirty and the alternative technologies. Similarly, for nations with a better quality of the environment, using the dirty technology at first is optimal, followed by, with investments in knowledge to reduce the backstop cost, eventually converging to a steady state of using the two technologies.

The optimal solution in our model is a feasible and realistic one given falling unit costs for alternative technologies (which are still significantly higher relative to conventional energy sources ${ }^{3}$ ), which have led to their wider use in some nations. We contrast the optimal solution with the equilibrium one when atomistic firms do not realize the external effects of their own actions on the aggregate stock of environmental quality. As a result, individual firms never invest in knowledge (or engage in R\&D activities) to reduce backstop cost, and we get the extreme result that a firm in equilibrium always sticks to the dirty technology. We show that in the presence of external costs of pollution from using the dirty technology, it is optimal to invest in the clean technology very early to help an economy reach a steady state of using both the dirty technology and the backstop. Although the economy can completely switch to only using the clean resource with more investments over time, switching to these relatively less efficient technologies (e.g., wind and solar energies face the huge burden of lack of good storage solutions) may entail a temporary drop in consumption at the time of switch (Boucekkine et al., 2004, 2011, 2013a). ${ }^{4}$

Rising demand for coal by the growing economies of China and India, and a sustained increase in demand from the United States, has been met with rising

2 Our idea of a falling average backstop cost with investments in knowledge follows Tsur and Zemel (2003).

3 The U.S. Energy Information Administration (EIA, 2014) estimates U.S. average levelized costs for electricity generation for plants entering service in 2019 (in $2012 \$ / \mathrm{MWh}$ ) to be 95.6 for conventional coal, 102.6 for biomass, and 130.0 for solar.

4 Boucekkine et al. (2003, 2011, 2013a) model cleaner technologies as having a lower marginal productivity of capital, and switching from relatively efficient dirtier technologies leads to capital "obsolescence" and a temporary fall in consumption. 
coal production from China, India, and Australia. ${ }^{5}$ With a possible increase in world oil prices and the use of enhanced oil recovery technologies, the EIA predicts the production of world petroleum and other liquid fuels (hydrocarbons) to increase by 28.3 million barrels per day between 2010 and 2040. Thus, given the relative inexhaustibility of essential fossil fuels such as coal and gasoline, switching to a steady state of using a constant mix of dirty and clean technologies for extended periods is feasible and would arrest harmful potential environmental damages to future generations. Dasgupta (2008) states that $\mathrm{CO}_{2}$ concentration in the atmosphere is 385 parts per million, which according to ice cores in Antarctica, is the highest level reached in the past 650,000 years. The author further states that given the nonlinearities governing the earth's ecological system, little action to avoid climate change may involve the planet crossing many irreversible tipping points. On the other hand, leaving some of the stock of dirty technologies in the ground translates to losses of millions of dollars of GDP and would lead to unemployment and significant political and social tensions. The problem of how soon to start investing in alternative technologies and the time of switch to using more of the backstop is no doubt an interesting problem (according to Stern [2007], substantial steps to reduce emissions must be taken right now to prevent global average temperatures increasing by more than the critical value of $5^{\circ} \mathrm{C}$ ).

Our work can be placed in the literature of regime switching by Boucekkine et al. (2004, 2011, 2013a, 2013b), Acemoglu et al. (2012), and other work by Tsur and Zemel $(2003,2005)$ in which switching from a dirty to a clean resource may involve a temporary drop in consumption because of shifting to a new regime of less efficient technology. ${ }^{6}$ Similar to the studies by Boucekkine et al. (2013a, 2013b), in which the backstop is adopted in the optimal solution to prevent further environmental damages after an "ecological threshold" (which implies a lower regeneration capacity of the earth) is reached, adoption of the clean technology in our model takes place to maintain environmental quality at a constant level or at times to improve it for a low endowment of environmental quality. One of the main differences between our work and that of Boucekkine et al. $(2004,2011,2013 \mathrm{a}, 2013 \mathrm{~b})$ is that we contrast the optimal and equilibrium solutions. We get a similar result to Acemoglu et al. (2012) of the behavior of

5 The EIA (2014) predicts China's coal production to increase from 3.5 billion short tons (bst) in 2010 to 4.1 bst in 2015 and to 5.7 bst in 2040. An estimate of world coal reserves provided by the German Federal Institute for Geosciences and Natural Resources (BGR, 2013) and the World Coal Association (2013) predicts approximately 1,038 billion tons (1,144.7 bst) of reserves left as of 2012, equivalent to 132 years of global coal output at the current rate.

6 Barbier (1999) and Schou (2000) include exhaustability of the dirty resource and find that when it is essential in production, optimal consumption paths would be declining and approach zero in the long term. Nondeclining consumption profiles in the steady state can be maintained by gradual substitution of the exhaustible resource by investments in human capital (similar to investments in physical capital by Stiglitz [1974a, 1974b] and Solow [1974]). 
individual firms in equilibrium leading to a severe deterioration of environmental quality ("environmental disaster" in Acemoglu et al. [2012]). In Boucekkine et al. (2004) and Acemoglu et al. (2012), a widening technological gap between the dirty and clean sectors causes environmental degradation; the former introduces a learning curve (where the economy learns about the new technology from the very beginning) to eliminate this efficiency gap, whereas the latter resorts to government intervention to help improve the productivity of the dirty sector. Although we attempt to characterize the difference between the optimal and equilibrium solutions and show how the optimal solution makes intuitive sense, efforts to bring the two solutions closer is something we leave for our future work. We discuss in the concluding remarks some practical correction mechanisms (for firms to internalize the externalities) and policy implications.

The paper is organized as follows: we provide our main model (the "Model" section); solve for special cases when we do not have knowledge accumulation and when knowledge accumulation is included to reduce the average cost of the backstop technology (the "Model without Knowledge Accumulation" and "Model with Knowledge Accumulation" sections, respectively); and discuss the key results and conclusions (the "Concluding Remarks" section).

\section{Model}

We consider a model consisting of a continuum of measure 1 of identical firms. Each firm is owned by an infinitely lived household. We abstract from population growth and for simplicity, and we normalize the total population in the economy to be unity.

A representative firm in the economy produces a "composite commodity" using a polluting natural resource and a backstop technology. The composite commodity is used for both consumption and investment. Energy, or the sum of the use of the polluting natural resource and the backstop, is regarded as the only input for a representative firm. We assume both the polluting resource and the backstop to be in nearly unlimited supply. We henceforth refer to the polluting resource as the "dirty technology" and the backstop as the "clean technology."

The costs of production for a firm are divided into the costs of the dirty and clean technologies. There is no cost of extraction for the dirty technology. The only cost associated with its use is the cost of pollution, which adversely affects the profits for a representative firm. On the other hand, the firm faces a positive average cost for the clean technology each period. However, continuous investments in knowledge taken out of the composite commodity can help reduce this cost over time. Knowledge can be thought of as the technical know-how to operate alternative clean technologies like wind and solar. Investments through R\&D may bring about more efficient techniques to use these technologies lowering their average costs. Alternative technologies may be thought of as having a higher embodied technical progress (Boucekkine et al., 2004) or to be 
more sophisticated. Increasing the knowledge base for an economy would thus help people operate them more efficiently. In this paper, we assume innovation in technical know-how to operate alternative technologies is developed in the lab (i.e., we assume that each firm has its own R\&D sector). They do not come about through using the clean technologies themselves. The production function for the composite commodity is then given by

$$
y=e^{\alpha}
$$

where $y$ denotes the composite commodity, $e$ denotes total energy use, ${ }^{7}$ and $0<$ $\alpha<1$. All firms are assumed price takers, and entry and exit are not permitted in the model. Finally, we take the price of the composite commodity $y$ to be constant at unity. Total energy production is in turn given by

$$
e=b+r
$$

where $b \geq 0$ and $r \geq 0$ represent use of the clean and the dirty technologies by a representative firm. Because of the clean and dirty technologies being perfect substitutes, energy use can come from either source. Although the dirty technology is assumed to be in unlimited supply, there is a feasible limit $\bar{r}$ that firms can extract in any time period given their production techniques. So we impose $r \leq \bar{r}$ as a feasible technology constraint. The previous specification is similar to the study by Acemoglu et al. (2012), in which "dirty" and "clean" inputs are used to produce the final good. A representative firm can invest in knowledge out of total production. The knowledge accumulation function is given by

$$
\dot{n}=a \sqrt{i}
$$

where $n$ denotes the stock of knowledge for a representative firm. The initial endowment of knowledge stock, $n_{0}$, is assumed to be positive. Each firm is endowed with the same initial stock of knowledge. The variable $a>0$ represents the investment parameter, and $i \geq 0$ denotes the investment each period for a firm. A concave knowledge accumulation function implies that it pays to invest little amounts every period rather than a lot in any one period. We impose a ceiling on $n$ after which investments in knowledge do not further reduce the average cost of the clean technology. We denote this maximal level of knowledge by $\bar{n}$. This can be thought of as a stage where the productivity of knowledge accumulation reduces to zero. Investment, being worthless, falls to zero beyond this point.

In our setup, we capture pollution through adverse effects on environmental quality. Environmental quality is denoted by $A$ in the model. We assume $A>0$

7 We implicitly assume that the composite commodity is produced using both energy and labor where $1-\alpha$ is the share of labor. Population (and labor) is assumed to be unity, and the wage rate equals 1 as well. 
for all $t$. Introducing the possibility of an environmental disaster when $A<0$ (Acemoglu et al., 2012) or an ecological threshold with two regimes for $A$ (Boucekkine et al., 2013b) could generate interesting results.

The initial stock of environmental quality is given by $A_{0}>0$. Environmental quality worsens due to use of the dirty technology by all firms but regenerates at a constant rate $\gamma>0$. We assume that there exists a pristine level of environmental quality denoted by $\bar{A}$. We can write the relevant equations as

$$
\dot{A}=(\bar{A}-A) \gamma-R
$$

and

$$
R=\int_{0}^{1} r_{j} d j
$$

where $r_{j}$ denotes resource use by the $j$ th firm, and $R$ stands for aggregate use of the dirty technology for the economy. We do not allow for heterogeneity in firm choices (larger firms using more of the dirty technology) and assume that each firm contributes equally to $R$. A representative firm in equilibrium treats $R$ as constant. Being one among an infinite number of firms, it ignores the effect of its own action on the deterioration of environmental quality. Each firm, however, faces a damage cost $(\bar{A}-A)^{2}$ each period from the deterioration of environmental quality. A damage cost can be thought of in terms of lower worker productivity or poor worker health affecting production for the firm. Because the dirty technology is in infinite supply and is free, firms would use the maximum feasible amount $\bar{r}$ each period. As the relatively expensive clean technology is never used, investment in knowledge would be zero in the equilibrium solution. The social planner would, however, fully internalize the negative external effect of aggregate resource use. Investment would be carried out in the backstop technology to make it cheaper and so as to make a switch to the clean technology. The average cost of the clean technology as a function of the aggregate knowledge level is given by

$$
C(N)=a_{0}-a_{1} N
$$

such that

$$
N=\int_{0}^{1} n_{j} d j
$$

where $n_{j}$ stands for knowledge stock of the $j$ th firm, and $N$ denotes the aggregate stock of knowledge in the economy. In a similar fashion to equation (5), we assume away heterogeneity in firm choices such that each firm would invest an equal amount in R\&D every period. $N$ also denotes the average knowledge stock for the representative firm, and because only the planner invests in knowledge in our model, the aggregate consistency condition $N=n$ is substituted in equation 
(6) for the optimal solution. The variables $a_{0}>a_{1}>0$ are the cost parameters of the model.

\section{Model without Knowledge Accumulation}

\subsection{Optimal Solution}

The externality in environmental pollution leads to a divergence between the equilibrium and the socially optimal solution. Here, we explore a model with no knowledge accumulation or zero investment for the social planner. That is to say, $a=0$ in equation (3). Because aggregate consistency implies $N=n$ from equation (7), the aggregate knowledge stock $N$ would not change as a result. So cost of the clean technology given by equation (6) would remain constant.

We begin with this case for the following reason: Because there is only one state variable, $A$, it helps us to solve the entire path of energy use with relative ease. Moreover, the method of solving the model and the solutions remain qualitatively the same even when knowledge accumulation is included. This model provides a good starting point for the more complex case of investments in knowledge reducing the average cost of the clean technology. Depending on the initial stock of environmental quality, we get a situation of only using $r$ or $b$ before switching to simultaneous use of the two energy sources from the time their marginal costs become equal. For the case when the planner uses only the dirty technology, we assume an interior solution for $r$ or $r<\bar{r}$. So imposing the aggregate consistency condition $R=r$ and assuming $N=n_{0}=1$, we can write the maximization problem for the social planner solution as

$$
\max \int_{t=0}^{\infty}\left((b+r)^{\alpha}-\left(a_{0}-a_{1}\right) b-(\bar{A}-A)^{2}\right) e^{-\rho t} d t
$$

subject to equation (4) where $\rho>0$ denotes the constant rate of discount, $b, r \geq 0$ and $A_{0}$ given. The maximization problem for the social planner in our model is similar to the social welfare function employed by Boucekkine et al. (2013b); in contrast to a concave utility function of consumption, in our model production of the composite commodity is nonlinear in total energy use. We choose to maximize net profits in the previous problem as this may be more realistic as an objective function for individual firms (or a social planner) in a perfectly competitive economy. In many situations, fossil fuels (ignoring their exhaustibility) and alternative technologies are perfect substitutes, and this linearity in total energy use gives rise to corner solutions (only resource or only backstop use). ${ }^{8}$ 
The current-valued Hamiltonian of the problem is then

$$
\begin{aligned}
H= & (b+r)^{\alpha}-\left(a_{0}-a_{1}\right) b-(\bar{A}-A)^{2}+\lambda((\bar{A}-A) \gamma-r)+\theta_{1} b \\
& +\theta_{2} r+\theta_{3}(\bar{r}-r)
\end{aligned}
$$

where $\lambda$ is the shadow price of environmental quality, and the $\theta_{i}$ 's denote the nonnegativity and the technology constraint on $b$ and $r$, with $\lambda, \theta_{1}, \theta_{2}, \theta_{3} \geq 0$. The necessary conditions for an optimal solution are

$$
\begin{gathered}
\frac{\partial H}{\partial b}=\alpha(b+r)^{\alpha-1}-\left(a_{0}-a_{1}\right)+\theta_{1}=0, \theta_{1} b=0 \\
\frac{\partial H}{\partial r}=\alpha(b+r)^{\alpha-1}-\lambda+\theta_{2}-\theta_{3}=0, \theta_{2} r=0, \theta_{3}(\bar{r}-r)=0 \\
\dot{\lambda}=\rho \lambda-\frac{\partial H}{\partial A}=(\rho+\gamma) \lambda-2(\bar{A}-A),
\end{gathered}
$$

and the transversality condition is given by

$$
\lim _{t \rightarrow \infty} e^{-\rho t} \lambda(t) A(t)=0 .
$$

Because the dirty and clean technologies are perfect substitutes in the previous model, they have equal marginal benefits. When only using the dirty technology $\left(0<r\left\langle\bar{r}, b=0 \Rightarrow \theta_{2}=0=\theta_{3}, \theta_{1}\right\rangle 0\right)$, its optimum path is given by

$$
e^{*}=r^{*}=\left(\frac{\alpha}{\lambda}\right)^{\frac{1}{1-\alpha}}
$$

and we have

$$
\lambda=\left(a_{0}-a_{1}\right)-\theta_{1} .
$$

Similarly, when only using the clean technology $\left(b>0, r=0 \Rightarrow \theta_{1}=0=\right.$ $\left.\theta_{3}, \theta_{2}>0\right)$, its optimum path is given by

$$
e^{*}=b^{*}=\left(\frac{\alpha}{a_{0}-a_{1}}\right)^{\frac{1}{1-\alpha}}
$$

and we have

$$
\lambda=\left(a_{0}-a_{1}\right)+\theta_{2} .
$$

So when using both the energy sources $\left(b>0,0<r<\bar{r} \Rightarrow \theta_{1}, \theta_{2}, \theta_{3}=0\right)$, we have the conditions

$$
e^{*}=\left(\frac{\alpha}{\lambda}\right)^{\frac{1}{1-\alpha}}=\left(\frac{\alpha}{a_{0}-a_{1}}\right)^{\frac{1}{1-\alpha}}
$$




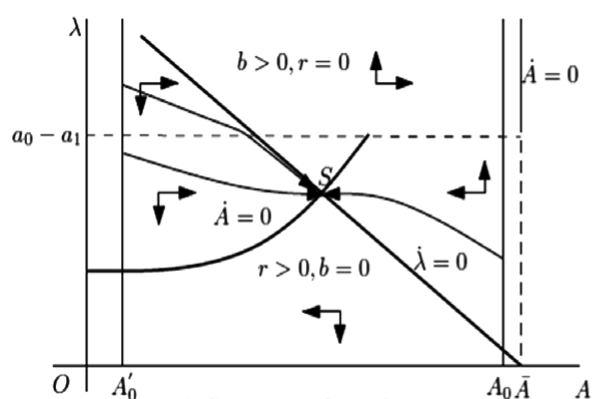

(a) Conventional steady state

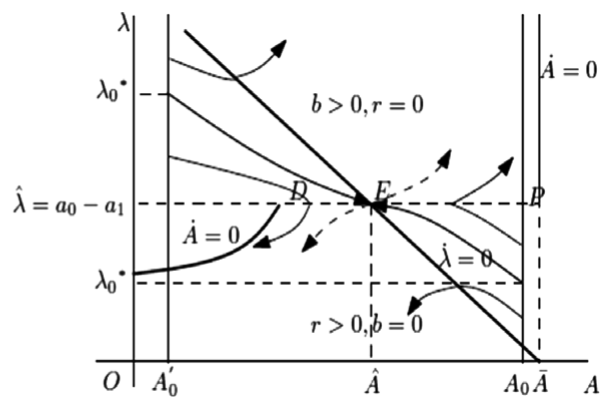

(b) Modified steady state

Figure 1. Conventional and Modified Steady State

and

$$
\lambda=\left(a_{0}-a_{1}\right) .
$$

It should be noted from the previous equations that total energy used would be constant in the case of simultaneous use of $r$ and $b$. However, their division is indeterminate.

Equation (18) is central to our analysis. Given a constant average cost of $b$ and a free but polluting natural resource, simultaneous use of the two energy sources is only possible when the shadow price of environmental quality rises or falls to equal $\left(a_{0}-a_{1}\right)$. That is to say, if $\lambda>0$, then we can get to a situation of simultaneous use of $r$ and $b$ from using any one of the energy sources at first. Equations (14) and (16) show that equation (18) can be satisfied starting from $\lambda$ less than or greater than $\left(a_{0}-a_{1}\right)$.

\section{Conventional and Modified Steady State: Solving for the Optimal Solution}

This section deals with solving for the optimal solution using a phase diagram analysis. We find motion trajectories that are feasible and satisfy the necessary conditions for optimality. To check for the sufficient condition, we look at the transversality condition given by equation (12). If we find paths that satisfy both these conditions, then we say such paths are optimal. We first define a "conventional steady state" and a "modified steady state" in this regard.

Figure 1 shows a stylized version of the stationary loci, the stable arms and the possible levels of environmental quality. In Figure 1a, the point of intersection of the stationary loci for equations (4) and (11) is referred to as the conventional steady state. It is denoted by point $S$. Given equation (18), Figure 1 shows that only the clean technology is used above the line $\left(a_{0}-a_{1}\right)$, the dirty technology below it, and any combination of $b$ and $r$ along the line. $A_{0}$ and $A_{0}^{\prime}$ denote possible initial given levels of environmental quality (we demonstrate these two cases in one graph for ease of reading; both cases are shown separately in the Appendix). For a constant average cost of the clean technology above the shadow price of environmental quality implied at the conventional steady state, we show 
stable arms approaching point $S$ from either its southeast or northwest. In this case, the transversality condition given by equation (12) is satisfied. When the initial stock of environmental quality is relatively high $\left(\right.$ at $\left.A_{0}\right)$, only the dirty technology is used along the stable arm until we approach $S$ in infinity. Because $\lambda$ is rising in this region, this means decreasing use of the dirty technology over time according to equation (13). However, for a lower endowment of environmental quality $A_{0}^{\prime}$, we may have two stable arms approaching the conventional steady state from its northwest. Depending on our choice of $\lambda_{0}$, we may have a sequence of using the clean and then the dirty technology or only the dirty technology until we approach $S$ in infinity. Because $A$ rises at a faster rate when $r=0$, the stable arm in the former case will be relatively flat and then steep as we approach the conventional steady state.

Intuitively, the magnitude of the shadow price of environmental quality relative to that of the constant average cost of the clean technology is what determines a planner's decision of whether to use only the dirty or only the clean technology. For a relatively pristine level of environmental quality at $A_{0}$, the value of its additional unit on lifetime utility $(\lambda)$ would be smaller relative to $\left(a_{0}-a_{1}\right)$. Hence, it would be economical for the planner to use only the dirty technology until $\lambda$ rises to the level of the constant average cost of the clean technology. From then onward, the planner would use a mix of the clean and the dirty technologies. The economy can stay at this steady state forever as the dirty technology is inexhaustible. Conversely, when environmental quality is poor to begin with at $A_{0}^{\prime}$, the present value of its marginal increment on lifetime utility may be greater than the average cost of the clean technology making it cheaper for the planner to use only the clean energy input. The planner may, however, switch to the dirty technology again if environmental quality improves much lowering its shadow price. The more interesting case involves when the line $\left(a_{0}-a_{1}\right)$ is below the conventional steady state. This situation is depicted in Figure $1 \mathrm{~b}$. We once again show two possible initial endowments of environmental quality, $A_{0}$ and $A_{0}^{\prime}$, in one graph for an easier demonstration. Here the stationary locus for equation (4) is truncated at $\lambda=\left(a_{0}-a_{1}\right)$ : because we only use the clean technology for any $\lambda>\left(a_{0}-a_{1}\right)$, the $\dot{A}=0$ locus will coincide with $A=\bar{A}$ when $R=r=0$ in equation (4). It can be shown that point $E$ can be made a steady state at which $\dot{\lambda}=\dot{A}=0$. We call point $E$ the modified steady state. Given $\dot{\lambda}=0$ at $E$, the motion of $A$ can be set equal to zero through an appropriate choice of $r$ at any point along the open interval DP. Because equation (18) holds along this interval, we would also get simultaneous use of the two energy inputs. The coordinates at point $E$ are denoted by $(\hat{A}, \hat{\lambda})$. The choice of $r$ that makes $\dot{A}=0$ along the interval $D P$ can be obtained from equation (4) and imposing the aggregate consistency condition $(R=r)$ as

$$
r^{*}=\gamma(\bar{A}-\hat{A}) .
$$


Note that $\hat{\lambda}=\left(a_{0}-a_{1}\right)$ at $E$. From equation (17), we can then obtain the path of the clean technology as a residual:

$$
b^{*}=e^{*}-r^{*}
$$

Although not proved yet, we will show that these are indeed the optimal paths of $r$ and $b$. Figure $1 \mathrm{~b}$ shows that the modified steady state $E$ can be approached from either its northwest or southeast as indicated by the motion trajectories. Depending on the given initial stock of environmental quality, Figure $1 \mathrm{~b}$ shows stable arms approaching $E$ from either of these directions. It can be shown that both these stable arms are unique and that they approach $E$ from either direction in finite time. The system would stay at $E$ forever, and that this satisfies transversality would be explained later in the paper. If we, however, allow the stable arms to proceed further, they will either divert to the southwest or northeast as shown by the dashed arrows.

If the system starts at a relatively pristine level of environmental quality, that is at $A_{0}$ near $\bar{A}$, then there exists an optimum initial $\lambda$ (or $\lambda_{0}^{*}$ ) such that the motion trajectory originating from $\left(A_{0}, \lambda_{0}^{*}\right)$ becomes perfectly horizontal at $E$ and then proceeds toward its southwest. $A$ falls and $\lambda$ rises along this stable arm until it hits the modified steady state. For a choice of $\lambda_{0}<\lambda_{0}^{*}$ given $A_{0}$, the system would follow a motion trajectory that would hit the $\dot{\lambda}=0$ locus at a point below $E$ and then proceed toward its southwest. The shadow price of environmental quality would then reach zero in finite time. If $\lambda_{0}>\lambda_{0}^{*}$, then the motion trajectory will hit a point on the open interval $D P$ toward the right of $E$ after which it proceeds toward its northeast. Similarly, if the system begins with poor environmental quality at $A_{0}^{\prime}$, then we can also find a unique $\lambda_{0}^{*}$ such that the stable arm originating from $\left(A_{0}^{\prime}, \lambda_{0}^{*}\right)$ becomes perfectly horizontal at $E$ and then proceeds toward its northeast. Any other choice of $\lambda_{0}$ would result in the motion trajectory intersecting the $\dot{\lambda}=0$ locus either above Eor toward the left of it on the open interval $D P$. The path would then either proceed toward the northeast or southwest of the modified steady state $E$. Given a stock of environmental quality at $A_{0}$, only $r$ would be used along the stable arm until it gets infinitesimally close to $E$. The growth of $\lambda$ along the stable arm implies decreasing use of the dirty technology by equation (13). However, at this point there is a jump in the control $r$ such that its path changes to equation (19). The path of $b$ is given by equation (20). Given that $\hat{A}$ is constant at the modified steady state $E$, equations (19) and (20) imply that $r^{*}$ and $b^{*}$ are both constants. In addition, because of a constant $\hat{\lambda}$, the transversality condition (equation 12) would be satisfied. Because such a plan of only using the dirty technology at first and then a mix of the clean and dirty technologies is feasible and satisfies the necessary and sufficient conditions for optimality, we say that such a plan is optimal. The system would finally settle at $E$ and stay there forever. Similarly, given $A_{0}^{\prime}$, only the clean technology $b$ would be used along the stable arm at 


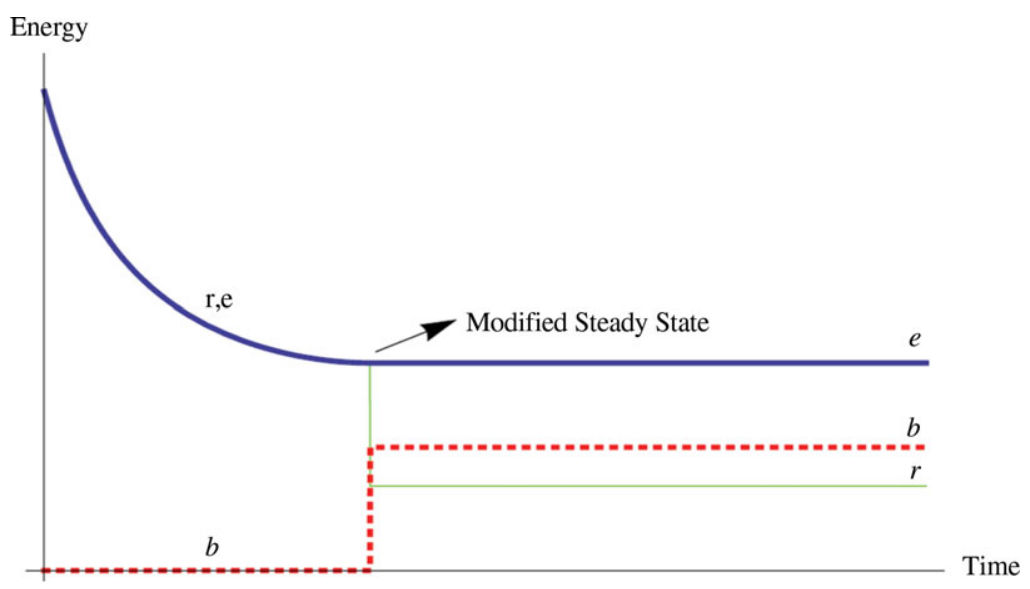

Figure 2. Energy Use Given Constant Average Cost of $b$

the constant rate given by equation (15) until it gets infinitesimally close to $E$. The path of $b$ then jumps to equation (20), and the path of $r$ jumps from $r^{*}=0$ to equation (19). We can then follow the previous analysis and show that the transversality condition (equation 12 ) would be satisfied. Such a plan would also be optimal.

Figure 2 shows the optimal paths for $e, b$, and $r$ over a long period of time if we begin at $A_{0}$. In Figure 2, the paths of $e$ and $r$ coincide when the planner only uses the dirty technology and gradually approaches the modified steady state. Because the shadow price of environmental quality $\lambda$ rises during this period, the path of $r$ follows a downward profile according to equation (13). As the clean technology is not used by the social planner, the path of $b$ coincides with the horizontal axis. At the modified steady state, total energy use becomes constant by equation (17), and $r$ jumps down to its new constant profile given by equation (19). So $b$ jumps up from $b^{*}=0$ to make up the difference: this is given by the constant path (equation 20). We solve for the optimal solution using numerical methods given the values of the parameters. As will be defined again later, we denote by $\hat{T}$ the time it takes for the stable arm to reach $E$ starting either from a relatively high or low initial stock of environmental quality. The Appendix provides the necessary numerical solutions.

Result 1: When a polluting resource and a clean alternative technology are perfect substitutes in producing a final good, an endowment of a relatively pristine environment implies that only the dirty technology is used by a social planner until a constant steady state is reached when the two technologies are used in fixed proportions. On the other hand, beginning from a relatively poor quality of the environment, the optimal solution involves only using the clean technology at first and then converging to the previously defined steady state. 


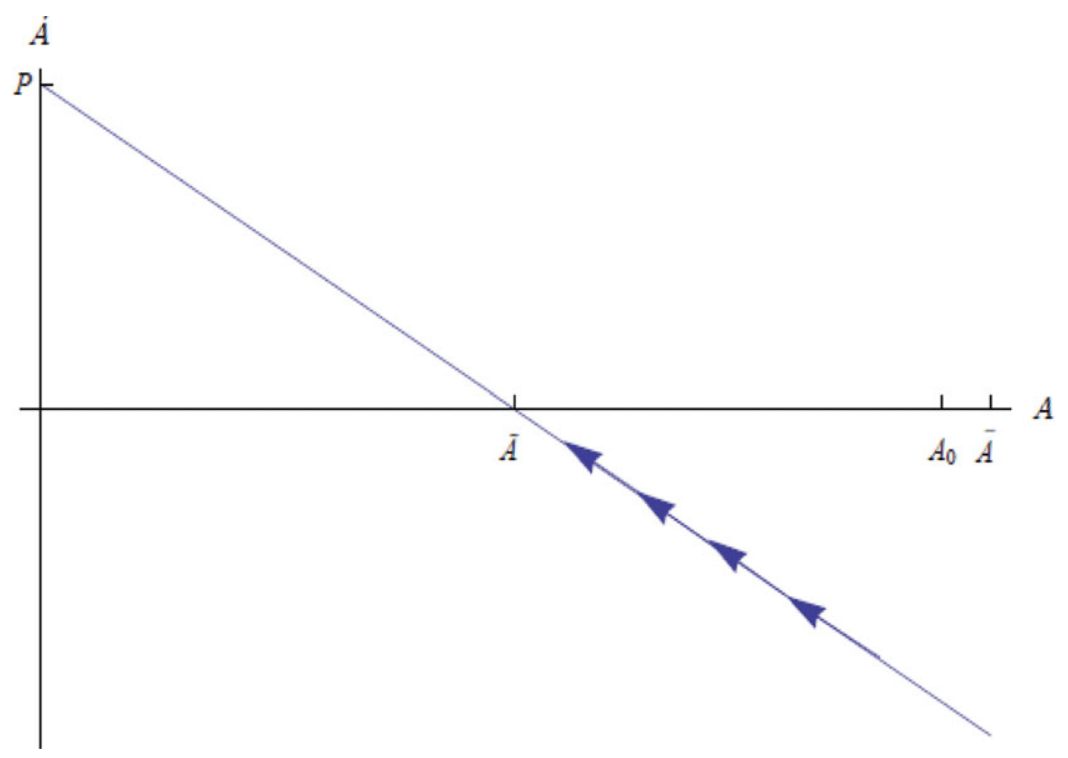

Figure 3. Transitional Dynamics of $A$ in Market Equilibrium

\section{Competitive Equilibrium Solution}

Individual firms in the market equilibrium select the cheaper dirty technologies ignoring their impact on the stock of environmental quality. Each firm treats $R$ from equation (4) as constant, and thus $\lambda$ becomes irrelevant in this case. Because a firm uses a constant amount $\bar{r}$ of the resource every period, the equilibrium problem also reduces to a static one. Investment in R\&D (to reduce average backstop cost) never occurs in the competitive equilibrium solution, and the problem would remain the same even when the model is extended to include knowledge accumulation.

The behavior of firms in equilibrium would, however, significantly reduce environmental quality. But a high enough constant rate of regeneration, $\gamma$, may stabilize environmental quality at some level $\tilde{A}$. Imposing $r=\bar{r}$ in equation (4), and setting $\dot{A}=0$, we get

$$
\tilde{A}=\bar{A}-\frac{\bar{r}}{\gamma} .
$$

Figure 3 shows the dynamics of $A$ for the equilibrium solution. A positive intercept in Figure 3 implies $\gamma>\frac{\bar{r}}{\bar{A}}$. We assume an $\bar{r}$ such that this inequality holds and $\tilde{A}>0$ (we rule out an $\bar{r}$ such that $\tilde{A}<0$ ). The arrows indicate the direction of movement to the stationary state $\tilde{A}$ if we start from a relatively high stock of environmental quality at $A_{0}$. Similarly, for a low initial stock of $A$, environmental quality would move down along the $\dot{A}$ locus to the stationary state $\tilde{A}$. Given the parameter values in the Appendix, it can be seen that $\tilde{A}<\hat{A}$. 


\section{Model with Knowledge Accumulation}

Here we solve the full model where investments in knowledge reduce the average cost of the clean technology. We start out with analyzing the optimal solution as in the previous model. For the case when the planner only uses the dirty technology, we assume $r<\bar{r}$. Furthermore, for simplicity, we impose $a=1$ in equation (3). Interestingly, similar results are obtained here as in the case when knowledge accumulation was not included: based on the endowment of environmental quality, the social planner would use only one energy source followed by the simultaneous use after a certain point in time.

Using the aggregate consistency conditions $(R=r)$ and $(N=n)$, we can write the maximization problem for the social planner solution as

$$
\max \int_{t=0}^{\infty}\left((b+r)^{\alpha}-\left(a_{0}-a_{1} n\right) b-i-(\bar{A}-A)^{2}\right) e^{-\rho t} d t
$$

subject to equations (3) and (4). Here $\rho>0$ denotes the constant rate of discount, $b, r, i \geq 0$ and $n_{0}$ and $A_{0}$ given. The current-valued Hamiltonian is then given by

$$
\begin{aligned}
& H=(b+r)^{\alpha}-\left(a_{0}-a_{1} n\right) b-i-(\bar{A}-A)^{2}+\mu \sqrt{i}+\lambda((\bar{A}-A) \gamma-r)+\theta_{1} b \\
& \quad+\theta_{2} r+\theta_{3}(\bar{r}-r)+\theta_{4} i
\end{aligned}
$$

where $\lambda$ and $\mu$ denote the shadow prices of stocks of environmental quality and knowledge. The $\theta_{i}$ 's have the same interpretation as before, with $\lambda, \mu, \theta_{i} \geq 0$. The first order conditions with respect to $r$ and $\lambda$ are given by equations (10) and (11). The other necessary conditions for an optimal solution are

$$
\begin{gathered}
\frac{\partial H}{\partial b}=\alpha(b+r)^{\alpha-1}-\left(a_{0}-a_{1} n\right)+\theta_{1}=0, \theta_{1} b=0 \\
\frac{\partial H}{\partial i}=-1+\frac{\mu}{2 \sqrt{i}}+\theta_{4}=0, \theta_{4} i=0 \\
\dot{\mu}=\rho \mu-\frac{\partial H}{\partial n}=\rho \mu-a_{1} b
\end{gathered}
$$

and the transversality conditions are given by equation (12) and

$$
\lim _{t \rightarrow \infty} e^{-\rho t} \mu(t) n(t)=0 .
$$

The previous necessary conditions imply that investment is always positive in the model. With the introduction of knowledge accumulation, equation (24) implies that the marginal benefit of investment for $i=0$ is infinity compared with a constant marginal cost of 1 . From equation (24), we also see that $\theta_{4}=-\infty$ 
when $i=0$, which is not possible. So substituting $\theta_{4}=0$ in equation (24), we get

$$
\dot{n}=\sqrt{i}=\frac{\mu}{2} \text {. }
$$

The optimum path for use of the dirty technology $(0<$ $\left.r\left\langle\bar{r}, b=0 \Rightarrow \theta_{2}=0=\theta_{3}, \theta_{1}\right\rangle 0\right)$ is given by equation (13) as for the case without knowledge accumulation. However, equation (14) now changes to

$$
\lambda=\left(a_{0}-a_{1} n\right)-\theta_{1} .
$$

When only using the clean technology $\left(b>0, r=0, \Rightarrow \theta_{1}=0=\theta_{3}, \theta_{2}>0\right)$, its optimal path is given by

$$
e^{*}=b^{*}=\left(\frac{\alpha}{a_{0}-a_{1} n}\right)^{\frac{1}{1-\alpha}},
$$

which implies

$$
\lambda=\left(a_{0}-a_{1} n\right)+\theta_{2} .
$$

So, when using both $r$ and $b\left(b>0,0<r<\bar{r}\right.$ and $\left.\theta_{1}, \theta_{2}, \theta_{3}=0\right)$, we have the following conditions

$$
e^{*}=\left(\frac{\alpha}{\lambda}\right)^{\frac{1}{1-\alpha}}=\left(\frac{\alpha}{a_{0}-a_{1} n}\right)^{\frac{1}{1-\alpha}}
$$

and

$$
\lambda=\left(a_{0}-a_{1} n\right) .
$$

The previous two equations are similar to those obtained for the case without knowledge accumulation. However because both $\lambda$ and $n$ change over time in the current model, the relative magnitudes of these variables at each point in time would be critical for satisfying the condition for simultaneous use given by equation (32). This would determine the time of switch from only using the dirty technology or the clean one to periods of simultaneous use of the two energy inputs.

\subsection{Solving for the Optimal Solution}

The method of solving for the optimal solution in the current model would be analogous to that followed in the model without knowledge accumulation. We employ a phase diagram to solve the model. The relevant figure (Figure 4) shows a stylized version of the modified steady state when knowledge accumulation is included for the current model. The average cost of the clean technology depicted by the line $\left(a_{0}-a_{1} n\right)$ will be henceforth referred to as the "cost line." Because $n$ rises with investments in knowledge, the cost line moves downward over time. 


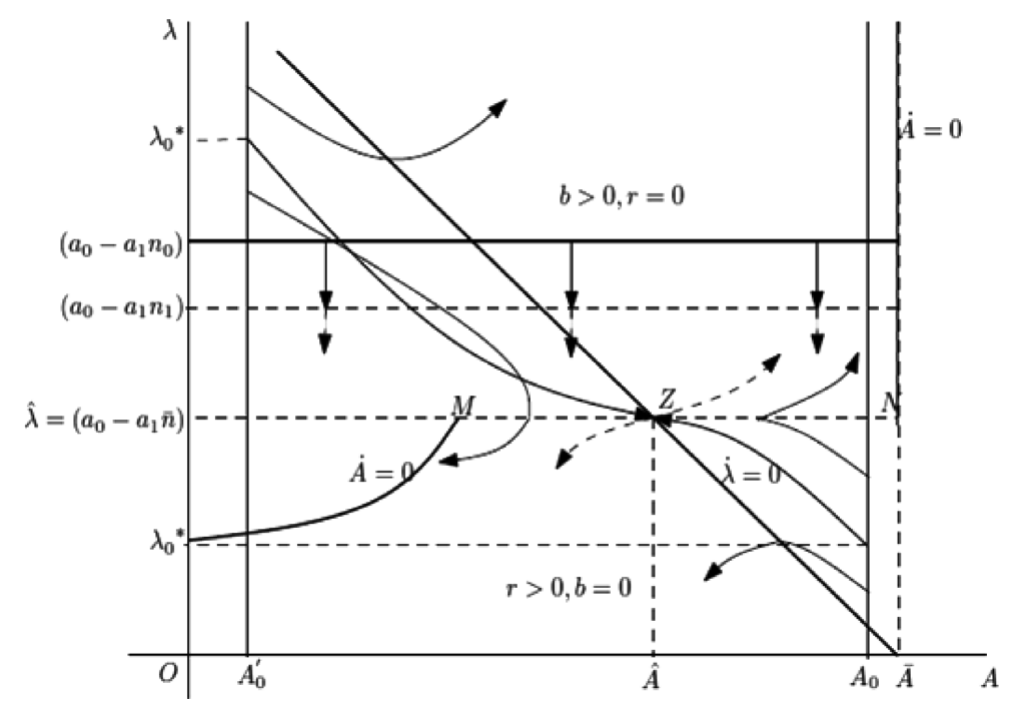

Figure 4. Transitional Dynamics of $(A, \lambda)$ with Investment in Knowledge

However as $n$ reaches its maximal level at $n=\bar{n}$, the cost line ceases to fall after it equals $\left(a_{0}-a_{1} \bar{n}\right)$. Investment becomes zero $(i=0)$ beyond this point, and the shadow price of the stock of knowledge $(\mu)$ becomes moot in this case. The intersection of the $\left(a_{0}-a_{1} \bar{n}\right)$ line with the $\dot{\lambda}=0$ locus (point $Z$ ) gives a possible steady state for the social planner solution. Given a significantly large $\bar{n}$ where the planner can possibly use the clean technology due to its low average cost, we assume the previous point of intersection $Z$ to be below point $S$ in Figure 1a. Following our logic in the previous model of an appropriate choice of $r$, we can make this point of intersection the modified steady state. The path of $r$ and $b$ would follow equations (19) and (20), respectively, at the modified steady state. We will return to this point when we fully explain the dynamics of the model. Note that the cost line would take a finite time to reach $\left(a_{0}-a_{1} \bar{n}\right)$ starting from its initial level at $\left(a_{0}-a_{1} n_{0}\right)$.

Figure 4 shows stable arms (policy functions) approaching the modified steady state $Z$ either from its southeast or northwest depending on the given initial stock of environmental quality. It is clear from the figure that the analysis with knowledge accumulation and with an upper bound on the stock of knowledge $\bar{n}$ is very similar to the previous model. However, the rate at which the cost line $\left(a_{0}-a_{1} n\right)$ falls over time will be key to our analysis. From equations $(25)$ and (27), this rate is given by $\mu$. In Figure 4, we see that given a relatively high or low initial stock of environmental quality, there exists an optimal choice of $\lambda_{0}$ such that the stable arm hits the falling cost line only at point $Z$ on the $\dot{\lambda}=0$ locus. We denote this optimal choice of $\lambda_{0}$ by $\lambda_{0}^{*}$. Note that at the modified steady state $Z, \hat{\lambda}, \hat{A}$, and $\bar{n}$ (by definition) are constants and that $\mu=0$. Because 
investment in knowledge falls to zero at $Z$ when $n=\bar{n}$, the shadow price of the knowledge stock $\mu$ jumps to zero from equation (27). So the transversality conditions given by equations (12) and (26) are satisfied at the modified steady state. The economy can stay at this steady state forever as the dirty technology $r$ is inexhaustible. We show that $\lambda_{0}^{*}$ is indeed the optimum such that given $A_{0}$, the stable arm originating from $\left(A_{0}, \lambda_{0}^{*}\right)$ intersects $\dot{\lambda}=0$ at the same time the cost line reaches $\left(a_{0}-a_{1} \bar{n}\right)$. The uniqueness of this stable arm can be illustrated by the following two cases:

1. $\lambda_{0}>\lambda_{0}^{*}$ : In this case, the motion trajectory would intersect the cost line toward the right of the stationary locus $(\dot{\lambda}=0)$. This would happen for $n<\bar{n}$ and in the special case of $n=\bar{n}$. Let us represent the time it takes for the motion trajectory to intersect the cost line as $t_{1}$. Because the cost line still keeps falling for $n<\bar{n}$, this implies that the motion trajectory would be above the cost line from the very next instant. The motion trajectory would then proceed to the northeast. For the knife-edge case of $n=\bar{n}$, any slight disturbance at $t_{1}$ would also take the motion trajectory to the northeast so that $\lambda$ becomes infinitely large.

2. $\lambda_{0}<\lambda_{0}^{*}$ : For an initial value of the shadow price of environmental quality below its optimal level, the motion trajectory would hit the $\dot{\lambda}=0$ locus at a point below $Z$ before the cost line reaches the level $\left(a_{0}-a_{1} \bar{n}\right)$. Let us call $t_{2}$ the time it takes for the motion trajectory to hit the stationary locus $\dot{\lambda}=0$. Then, from the next instant $t_{2}+\varepsilon$, the motion trajectory would proceed to the southwest and intersect the horizontal axis in finite time.

For a relatively low initial stock of environmental quality at $A_{0}^{\prime}, \lambda_{0}^{*}$ has to be significantly above $\left(a_{0}-a_{1} n_{0}\right)$ or the initial cost of the clean technology. This point deserves special mention. In this case, the policy function "catches" the falling cost line from above only at the point $Z$. For any lower level of $\lambda_{0}$, the motion trajectory may cross the falling cost line soon enough at a level of $n<\bar{n}$ after which the point on the trajectory will find itself below the cost line. The trajectory would then head to the southwest. The only way the motion trajectory can turn around and proceed back to the southeast toward $Z$ is if it intersects the falling cost line again and the cost line falls at a rate quicker than the point on the trajectory. In this case, the point on the trajectory would again be above the falling cost line. The uniqueness of $\lambda_{0}^{*}$ in this case can be proved in a similar way to the case for a relatively high stock of environmental quality. For $\lambda_{0}>\lambda_{0}^{*}>$ $\left(a_{0}-a_{1} n_{0}\right)$, the motion trajectory would intersect the $\dot{\lambda}=0$ locus sooner than the cost line reaches the level when $n=\bar{n}$. The motion trajectory would then proceed to the northeast in such a way that the shadow price of environmental quality would tend to an infinitely large value. For $\left(a_{0}-a_{1} n_{0}\right)<\lambda_{0}<\lambda_{0}^{*}$, the motion trajectory would hit a point to the left of the modified steady state $Z$ on the cost line when it falls to its minimal level at $\left(a_{0}-a_{1} \bar{n}\right)$. From the very next instant, the time path would proceed to the southwest and intersect the horizontal axis in finite time. 
So, if we begin with a stock of environmental quality at $A_{0}$, only the dirty technology is used according to equation (13) until the stable arm gets infinitesimally close to $Z$. This is evident from Figure 4. At $Z, r$ jumps to its new profile given by equation (19). The path of the clean technology is then obtained from the residual of equations (31) and (19) with $n$ replaced by $\bar{n}$. Similarly, for a relatively low initial stock of environmental quality at $A_{0}^{\prime}$, the planner would only use the clean technology along the stable arm as long as it is above the falling cost line. Because they only intersect at the modified steady state $Z$, the path of $b$ would be given by equation (29) until the policy function gets infinitesimally close to $Z$. At this point, $r$ jumps from $r^{*}=0$ to its new path given by equation (19). Once again, the path of the clean technology is obtained from the residual of equations (31) and (19).

We now deal with the optimal choice of $\mu_{0}$ given the unique $\lambda_{0}^{*}$ found previously for a high or low initial stock of environmental quality. Given $\left(A_{0}, \lambda_{0}^{*}\right)$ or $\left(A_{0}^{\prime}, \lambda_{0}^{*}\right)$ and using equations (4) and (11), we can find the time that it takes the stable arm to reach $Z$. We denote this time as $\hat{T}$. Given $\hat{T}$, there is a unique $\mu_{0}$ that will drive the cost line down from $\left(a_{0}-a_{1} n_{0}\right)$ to $\left(a_{0}-a_{1} \bar{n}\right)$ in exactly $\hat{T}$ years.

We refer to this optimal choice of $\mu_{0}$ as $\mu_{0}^{*}$. To verify, we can use $\mu_{0}^{*}$ to find the time $\bar{T}$ it takes for the cost line to reach $\left(a_{0}-a_{1} \bar{n}\right)$ starting from $\left(a_{0}-a_{1} n_{0}\right)$. Given $\hat{A}$, we can assume either $A_{0}$ to be less or greater than $\hat{A}$ (or $A_{0}^{\prime}$ to be less or greater than $\hat{A}$ ). We can then find a $\hat{\lambda}_{0}$ such that a motion trajectory originating from $\left(A_{0}, \hat{\lambda}_{0}\right)$ or $\left(A_{0}^{\prime}, \hat{\lambda}_{0}\right)$ hits the cost line when $n=\bar{n}$ in exactly $\bar{T}$ years. If $\hat{\lambda}_{0}$ equals $\lambda_{0}^{*}$ found previously, we can say that there exists a unique $\mu_{0}^{*}$ given $\lambda_{0}^{*}$. Numerical solutions for the optimal initial values of the costates and the time it takes for the stable arms to reach the modified steady state $Z$ are provided in the Appendix.

A few comments should be noted here. The optimal solution in this more realistic case when knowledge accumulation reduces the average cost of the clean technology produces the result that the dirty and clean energy inputs are used in fixed proportions in the steady state (similar to the previous case without investment in knowledge). It is optimal for the planner to always invest in knowledge (investments in knowledge are always positive), a result that comes from the concavity of equation (3). Introducing learning by doing in the knowledge accumulation function would add convexity to equation (3), and the stock of knowledge at the point where returns to investment change from increasing to diminishing might correspond to some critical knowledge stock. ${ }^{9}$ It would be interesting to see if this critical stock of knowledge coincides with the time of switch from only using the dirty or clean energy input to a constant mix of the two sources. The specification of equation (3) implying diminishing

9 Boucekkine et al. (2004) call a critical knowledge stock $A^{*}$ when introducing a learning curve. 
returns to knowledge accumulation also leads to one of the main conclusions of the paper: the dirty and clean technologies are used in fixed proportions in the steady state. The steady state corresponds to the stock of knowledge $\bar{n}$ (we assume this to be the maximum knowledge stock where returns to investment falls to zero for all practical purposes), and total energy use becomes constant from equation (31). It is intuitive that with diminishing returns to knowledge accumulation and a changing stock of environmental quality (because of either using $r$ or $b$ ), the marginal costs of the two technologies would become equal at some point. Our model shows that the marginal costs become equal at the steady state when environmental quality settles at a constant level and the marginal cost of the clean technology falls to its minimum at $\left(a_{0}-a_{1} \bar{n}\right)$. With a constant level of environmental quality, use of the dirty resource would be constant; use of the backstop obtained as a residual from total energy use is also contant as a result. There are no limits to how much knowledge the planner can accumulate at a point in time, and a faster than optimal knowledge accumulation may entail the cost line falling to $\left(a_{0}-a_{1} \bar{n}\right)$ before the policy function originating from a high or low endowment of environmental quality reaches that level. The policy function would then head to the northeast or southwest, and from our previous analysis, a modified steady state would not be attained as a result.

We next turn to the competitive equilibrium for the previous model with knowledge accumulation. The solution would look exactly like the one for the case without knowledge accumulation. A representative firm would always only use the dirty technology and extract the maximum feasible amount $\bar{r}$ every period. The relatively expensive clean technology would never be used, and because $i=0$, the stock of knowledge would remain at its initial level of $n_{0}$. The costate variables for the model, that is $\lambda$ and $\mu$, would both be irrelevant in this case. As in the model without knowledge accumulation, environmental quality may stabilize at some level $\tilde{A}$ given a sufficiently high regeneration rate. The dynamics of $A$ are then given in Figure 3. Once again, the economy can reach the stationary state starting from a high or low initial stock of environmental quality.

Result 2: The social planner solution remains qualitatively the same when knowledge accumulation (to reduce the average cost of the backstop) is included. The main difference from the previous model is that gradual investments in the backstop from the very beginning help the planner to attain the steady state of dual use of the two technologies when their marginal costs are equal. This steady state corresponds to a very high knowledge stock such that investments in the backstop are no longer worthwhile (i.e., the marginal benefit of investing an extra unit in the backstop falls to zero). Depending on the endowment of environmental quality, the steady state can be reached from either using the dirty or clean technology at first. 


\section{Concluding Remarks}

There is a significant gap in our model between the optimal (planner) and equilibrium solutions to the basic problem of using the dirty technology. Individual agents (firms) in equilibrium cause a greater deterioration of environmental quality because they overuse the dirty resource. A representative firm treats environmental quality as constant and ignores its own impact in reducing the stock of environmental quality. We show that a representative firm always sticks to the dirty technology and never invests in R\&D to reduce the average cost of the clean alternative technology (backstop). The social planner on the other hand internalizes the environmental externality (from use of the dirty technology), invests in R\&D to reduce the backstop cost, and switches to a mix of using the clean and dirty technologies in the steady state. The model is simple enough that it arrives at a key conclusion of adopting the backstop in a steady state to prevent further environmental degradation but without introducing any "ecological threshold" (Boucekkine et al., 2013b). ${ }^{10}$ Secondly, a comparison of the optimal and equilibrium solutions is made (as in Acemoglu et al., 2012) in an event where the market solution necessarily leads to a severe deterioration of environmental quality. Last but not the least, our paper introduces knowledge accumulation improving technical know-how in clean technologies (reducing their cost) into the literature of regime switching. Knowledge accumulation features diminishing returns in our model, which seems to be a realistic assumption.

In our model, we assume there is a separate $R \& D$ sector within each firm that is devoted to improving the technical know-how about alternative technologies (e.g., storage solutions for solar and wind and better fracking methods to reduce groundwater contamination when extracting natural gas) and not a sector where innovations come about through learning by doing. Introducing learning by doing would generate interesting possibilities in a way that knowledge accumulation such as in equation (3) would first be convex and then concave (when diminishing returns set in). In our current model, introducing learning by doing and adding firm heterogeneity (larger firms taking better advantage of learning by doing) may enable larger firms to switch sooner to the clean technology as compared with smaller firms. Further, a situation may arise when equality of the marginal costs of the two technologies occurs when the returns to investment in knowledge by the planner are still increasing; because of continually falling costs of the clean technology, a steady state in this case may correspond to only using the clean technology when returns to investment fall

10 In Boucekkine et al. (2013b), the cleaner technology is adopted after crossing the ecological threshold. 
approximately to zero. Environmental quality would then settle at a constant level in the steady state. ${ }^{11}$

Earlier work by Bovenberg and Smulders (1996), Grimaud and Rougé (2005), and Acemoglu et al. (2012) prescribes some correction mechanisms to bring the equilibrium solution closer to the optimal one when an environmental externality exists from using the dirty resource. ${ }^{12}$ In our paper, with a continuum of identical firms, a policy to make an individual firm internalize some of the pollution costs from using the dirty technology would be to impose a tax on its use. Another mechanism to bring the equilibrium solution closer to the planner one are giving research subsidies to firms (similar to Acemoglu et al. [2012]) to encourage investment in new knowledge. Patent rights' could then be added preventing knowledge spillovers (maintaining our continuum of identical firms assumption). Finally, cap-and-trade policies (allocating each firm some carbon credits and allowing trade in these credits) is another possibility if firm heterogeneity is added to our model. In this case, a result may be that larger firms invest more in the backstop and switch sooner than smaller firms; we leave this for future work.

\section{References}

Acemoglu, D., P. Aghion, L. Bursztyn, and D. Hemous. "The Environment and Directed Technical Change." American Economic Review 102,1(2012):131-66.

Barbier, E.B. "Endogenous Growth and Natural Resource Scar-ci-ty." Environmental and Resource Economics 14,1(1999):51-74.

Boucekkine, R., J. Krawczyk, and T. Vallée. "Environmental Quality versus Economic Performance: A Dynamic Game Approach.” Optimal Control Applications and Methods 32,1(2011):29-46.

Boucekkine, R., A. Pommeret, and F. Prieur. "Technological vs. Ecological Switch and the Environmental Kuznets Curve." American Journal of Agricultural Economics 95,2(2013a):252-60.

_. "Optimal Regime Switching and Threshold Effects." Journal of Economic Dynamics and Control 37,12(2013b):2979-97.

Boucekkine, R., C. Saglam, and T. Vallée. "Technology Adoption under Embodiment: A TwoStage Optimal Control Approach.” Macroeconomic Dynamics 8,2(2004):250-71.

Bovenberg, A.L., and S.A. Smulders. "Transitional Impacts of Environmental Policy in an Endogenous Growth Model.” International Economic Review 37,4(1996):861-93.

Dasgupta, P. "Discounting Climate Change." Journal of Risk and Uncertainty 37,23(2008):147-69.

Dasgupta, P., and G.M. Heal. “The Optimal Depletion of Exhaustible Resources.” Review of Economic Studies 41(1974):3-28.

11 We think the optimal solution would involve using only the dirty or clean energy input at first (depending on the endowment of environmental quality), then a mix of the two technologies at one point in time when their costs are equal, and then use of only the clean technology (similar to Hung and Quyen [1993] who have resource exhaustibility but do not include pollution).

12 Bovenberg and Smulders (1996) introduce an exogenous pollution ceiling implemented through a pollution tax. 
German Federal Institute for Geosciences and Natural Resources (BGR). Internet site: http://www.bgr.bund.de (Accessed June 15, 2014).

Grimaud, A., and L. Rougé. "Polluting Non-Renewable Resources, Innovation and Growth: Welfare and Environmental Policy." Resource and Energy Economics 27(2005):10929.

Hung, N.M., and N.V. Quyen. "On R\&D Timing under Uncertainty: The Case of Exhaustible Resource Substitution.”Journal of Economic Dynamics \& Control 17,5-6(1993):97191.

Schou, P. "Polluting Non-Renewable Resources and Growth." Environmental and Resource Economics 16,2(2000):211-27.

Solow, R.M. "Intergenerational Equity and Exhaustible Re-sour-ces." Review of Economic Studies 41(1974):29-45.

Stern, N. The Economics of Climate Change: The Stern Review. New York: Cambridge University Press, 2007.

Stiglitz, J. "Growth with Exhaustible Natural Resources: Efficient and Optimal Growth Paths." Review of Economic Studies 41(1974a):123-37.

. "Growth with Exhaustible Natural Resources: The Competitive Economy." Review of Economic Studies 41(1974b):139-52.

Tsur, Y., and A. Zemel. "Optimal Transition to Backstop Substitutes for Nonrenewable Resources.” Journal of Economic Dynamics \& Control 27,4(2003):551-72.

- "Scarcity, Growth and R\&D." Journal of Environmental Economics and Management 49,3(2005):484-99.

U.S. Energy Information Administration (EIA). “Annual Energy Outlook.” Internet site: http://www.eia.gov/forecasts/aeo (Accessed June 15, 2014).

World Coal Association. Internet site: http://www.worldcoal.org/resources (Accessed June 15, 2014).

\section{Appendix}

We use the parameter values $\alpha=0.33, a_{0}=10, a_{1}=0.002, \bar{A}=2, \bar{r}=0.017$, $A_{0}=1.99, A_{0}^{\prime}=0.8, n_{0}=0.01, \bar{n}=1,000, \gamma=0.01$, and $\rho=0.04$. We use the program Mathematica to compute numerical solutions for the model when knowledge accumulation is not included and when investments in knowledge reduce average backstop cost.

Based on the previous parameter values, we find the coordinates of $S$, or the conventional steady state in Figure $1 \mathrm{a}$, are $A_{S}=1.64$ and $\lambda_{S}=14.35$. For the model without knowledge accumulation, Figure A1 shows the two possible cases when the stock of environmental quality begins at a relatively high or low level. The coordinates of the modified steady state $E$ in Figure $A 1$ are $\hat{A}=1.75$ and $\hat{\lambda}=$ 9.998 given the previous parameter values. We find the stable arms using numerical methods and by working our way backward. For the equilibrium solution, $\tilde{A}=0.3$.

A similar analysis would follow when investments in knowledge are included to reduce the average cost of the clean technology. Figure A2 shows the stable arms for the two cases when an economy is endowed with a relatively high or low stock of environmental quality. We employ numerical methods to find these stable arms. The coordinates of the modified steady state $Z$ in Figure $A 2$ are $\hat{A}=1.8$ and $\hat{\lambda}=8$. The point of intersection of the stable arms with the initial stock of $A$ gives us a unique 
$\lambda_{0}^{*}$. We find $\mu_{0}^{*}$ using equations (25), (27), and (29) such that the cost line $\left(a_{0}-a_{1} n\right)$ falls to $\left(a_{0}-a_{1} \bar{n}\right)$ at the same time the stable arms reach $Z$.

Table A1 summarizes the numerical solutions of the two models when the average cost of the clean technology is constant and when it can be reduced through investments in the stock of knowledge. Given the previous parameter values, we notice some significant differences based on the initial stock of $A$ for each of the two models. For both the models, when the planner only uses the dirty technology for a high initial level of $A$, it reaches the modified steady state relatively soon compared with only using the clean technology given $A_{0}^{\prime}$. A high $\hat{T}$ implies greater investments from an early period or a high initial shadow price of knowledge $\mu_{0}^{*}$. So $\mu_{0}^{*}$ would be smaller for a relatively low endowment of environmental quality.

Table A1. Optimal Values When Only $r$ or $b$ Is Used for the Two Models

\begin{tabular}{lll}
\hline \hline Model & Without Knowledge Accumulation & With Knowledge Accumulation \\
\hline$A_{0}($ only $r)$ & $\lambda_{0}^{*}=5.71, \hat{T}=36.94$ & $\lambda_{0}^{*}=5.48, \hat{T}=20.71, \mu_{0}^{*}=62.01$ \\
$A_{0}^{\prime}($ only $b)$ & $\lambda_{0}^{*}=40.0007, \hat{T}=156.88$ & $\lambda_{0}^{*}=40.0002, \hat{T}=179.18, \mu_{0}^{*}=0.062$ \\
\hline \hline
\end{tabular}
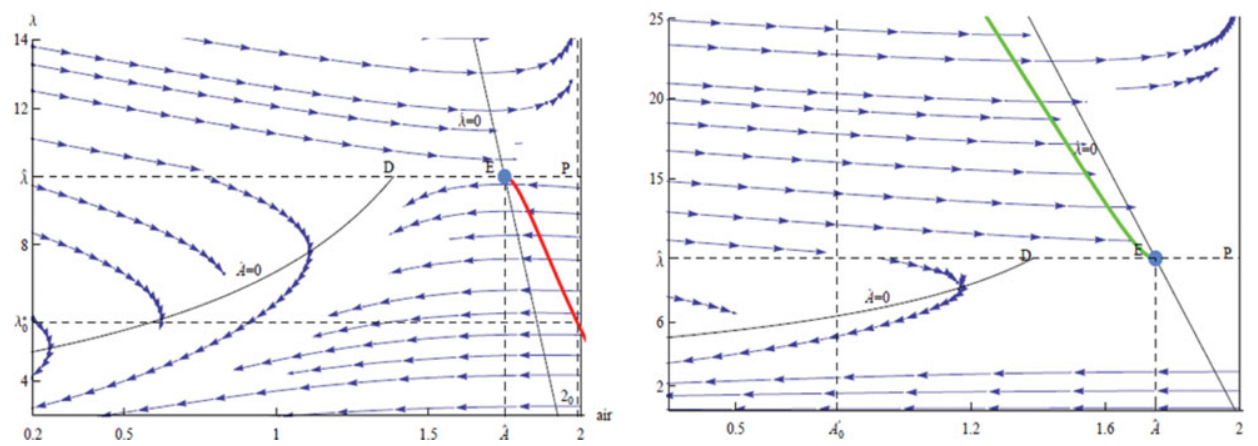

Figure A1. Transitional Dynamics of $(A, \lambda)$ with No Knowledge Accumulation
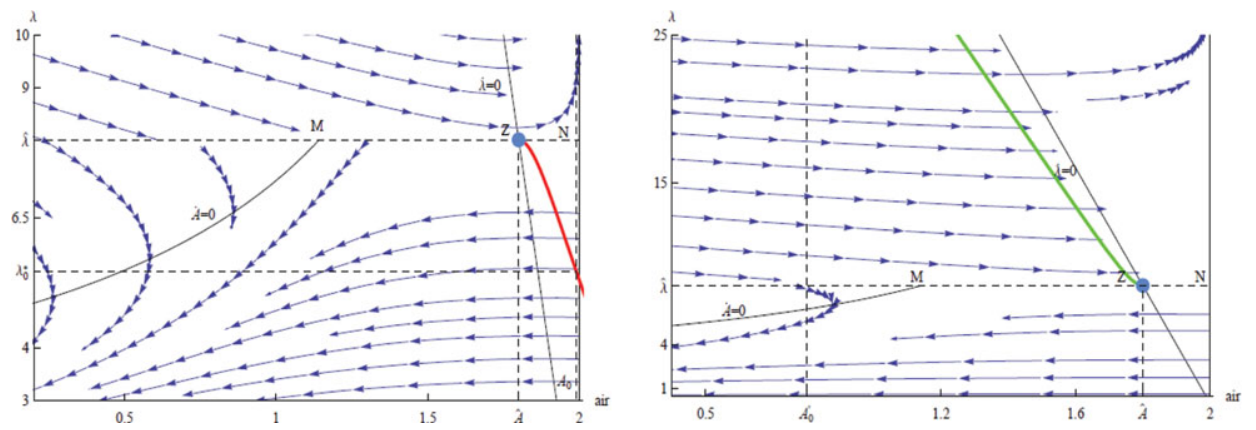

Figure A2. Transitional Dynamics of $(A, \lambda)$ with Investment in Knowledge 